\begin{tabular}{|c|c|}
\hline Title & Electrical treeing: A phase field model \\
\hline Author(s) & Cai, Ziming; Wang, X iaohui; Li, Longtu; Hong, Wei \\
\hline Citation & $\begin{array}{l}\text { Extreme mechanics letters, 28, 87-95 } \\
\text { https://doi.org/10.1016/.eml.2019.02.006 }\end{array}$ \\
\hline Issue Date & 2019-04 \\
\hline Doc URL & http:/hdl.handle.net/2115/79673 \\
\hline Rights & $\begin{array}{l}\text { () 2019. This manuscript version is made available under the CC-BY-NC-ND } 4.0 \text { license } \\
\text { https://creativecommons.org/icenses/by-nc-nd/4.0/ }\end{array}$ \\
\hline Rights(URL) & https:/creativecommons.org/icenses/by-nc-nd/4.0/ \\
\hline Type & article (author version) \\
\hline File Information & manuscript-electric-treeing-WH-19-2-18-no-mark.pdf \\
\hline
\end{tabular}

Instructions for use 


\title{
Electrical treeing: a phase-field model
}

Ziming Cai ${ }^{\mathrm{a}, \mathrm{d}}$, Xiaohui Wang ${ }^{\mathrm{a}}$, Longtu Li ${ }^{\mathrm{a}}$, Wei Hong ${ }^{\mathrm{b}, \mathrm{c}, \mathrm{d} *}$

${ }^{a}$ State Key Laboratory of New Ceramics and Fine Processing, School of Materials Science and Engineering, Tsinghua University, Beijing 100084, PR China

b Department of Mechanics and Aerospace Engineering, Southern University of Science and Technology, Shenzhen, Guangdong 518055, PR China

${ }^{\mathrm{c}}$ Global Station for Soft Matter, Global Institution for Collaborative Research and Education, Hokkaido University, Sapporo, Japan

${ }^{\mathrm{d}}$ Department of Aerospace Engineering, Iowa State University, Ames, IA 50010, USA

\section{Corresponding Author}

*Wei Hong, E-mail: hongw@sustc.edu.cn

\begin{abstract}
Electrical treeing is a commonly observed phenomenon associated with dielectric breakdown in solid dielectrics. In this work, a phase-field model is developed to study the initiation and propagation of electrical trees in two different geometries: a parallel capacitor and a cylindrical capacitor. The model utilizes a continuous field of damage variable to distinguish the localized damaged regions from the undamaged bulk material. Factors that affect electrical tree morphology, including discharge speed and damage mobility, are studied. Electrical treeing tends to exhibit a fractal shape with more branches at a relatively high discharge speed relative to the rate of damage. Furthermore, the effect of fillers on electrical treeing is also studied. Numerical results
\end{abstract}


suggest that fillers of lower permittivity, higher breakdown energy or lower damage mobility will promote fractal treeing and thus resist catastrophic breakdown.

Keywords: electrical treeing, dielectric breakdown, discharge speed, filler effect

\section{Introduction}

The idea of sustainable development has promoted the rapid growth of renewable energy industries in the past decade. As a result, high-efficiency energy-storage technologies are in high demand[1-5]. High-voltage dielectric materials (HVDMs) play an essential role in these technologies, since energy-storage devices like capacitors and inverters are often operated under high voltages[6-10]. Additionally, HVDMs have been used in many other industrial applications such as electric vehicles, high voltage transmission, kinetic energy weapons, and pulse-power circuits[11-13]. Unfortunately, dielectric breakdown often occurs in dielectric solids and limits the application of HVDMs[14, 15]. Electrical treeing often occurs as a precursor of most dielectric breakdown phenomena in $\operatorname{HVDMs}[16,17]$. The understanding of electrical treeing mechanisms is a key to the design and fabrication of new, reliable and higher-voltage dielectric materials. In theory, electrical trees initiate from the points of highly concentrated electric field[18]. Such an electric field localization may be cause by defects (e.g. voids and contaminants) or electromechanical fatigue due to the Maxwell stress[16], and are often accompanied by charge-carrier injection from electrodes and gas discharge caused by dielectric degradation. Compared with initiation, the growth 
and propagation of electrical trees are more dangerous since the breakdown happens when the gap between the electrodes is bridged by the electrical trees[19]. Attentions have been paid to study the propagation of electrical trees, especially that under an alternating current (AC) condition, and it has been reported that electrical tree propagation is controlled by many factors, such as the applied voltage, electric field enhancement, frequency, temperature and partial discharge[20-22]. Electrical trees tend to exhibit fractal patterns with more branches under relatively high applied voltages[23], temperatures[24, 25] or frequencies[26, 27]. In the last few decades, a number of models were proposed for the understanding of the phenomena[15, 16]. The models can be divided into two main categories, namely, statistical models and physics-based models. Statistical models use statistical analysis to describe the failure of dielectric solids by fitting experimental data to statistical distributions, including Weibull distribution[28], log-normal distribution[29], and Johnson SB distribution[30]. Different form statistical models, physics-based models include the mechanisms of one or more physical processes, such as the dielectric breakdown model[31, 32], the discharge-avalanche model[33], and the field-driven tree-growth model[34]. Pietronero et al.[32] proposed that dielectric breakdown processed are stochastic, and the growth of an electrical tree is not just governed by the local electric field but over the points of the highest probability of growing. Dissado et al.[33, 35] describe the electrical tree propagation through a discharged-avalanche model. However, the model requires some parameters, such as the characteristic time and the fractal dimension, which are hard to measure in practice. 
While existing models have brought some understanding to the mechanism of electrical treeing, the simulation of tree propagation is based mainly on statistical models or by reconstructing the intricate microscopic details. In this work, inspired by the similarity between dielectric breakdown and brittle fracture[36-39], a phase-field model is introduced to simulate the initiation and propagation of electrical treeing. The direct handling of the microscopic details in the process zone can be avoided by employing a Griffith-type energy criterion of the propagation of an electrical tree. Advantageously, the evolution of electrical trees and final breakdown pathways can be visualized through this model and the nominal breakdown strength can be calculated as well.

Similar to the mechanical toughening mechanism of composite materials, plenty of research has been carried out by using fillers to inhibit electrical treeing[40-48]. Various properties of these fillers can be modified, and the electrical treeing process can be altered. A more tortuous or branched breakdown path can dissipate more energy and therefore enhance the breakdown strength of dielectric materials[3]. In this contribution, dielectric composites filled with particles of different permittivity, breakdown energy, and damage mobility are also simulated to explore possible design strategies of highvoltage dielectric composites.

\section{Phase field model for electrical treeing}

The simulation on the initiation and propagation of electrical trees follows closely the phase-field model developed in Refs. $[49,50]$, by drawing an analogy between 
dielectric breakdown and mechanical fracture. A scalar phase field $s(\mathbf{x}, t)$ is introduced to characterize the electrical treeing in solid dielectrics. The value of $s$ varies continuously from the intact state, $s=1$, to the fully damaged state, $s=0$. The dielectric material becomes conductive locally at the fully damage region, but such a transition is difficult to capture numerically. In practice, a large but finite permittivity $\varepsilon_{0} / \eta$ is taken in the damaged region, with $\varepsilon_{0}$ being the initial permittivity and $\eta$ a sufficiently small number. At any other intermediate state, the permittivity of the solid dielectric is interpolated by

$$
\varepsilon(s)=\frac{\varepsilon_{0}}{f(s)+\eta}
$$

where $f(s)=4 s^{3}-3 s^{4}$. From a thermodynamic perspective, the criterion for electrical tree propagation is interpreted as: material damage takes place if the process decreases the total potential energy of the system

$$
\Pi[s, \phi]=\int_{\Omega}\left[W_{\mathrm{es}}(\mathbf{E}, s)+W_{\mathrm{d}}(s)+W_{\mathrm{i}}(\nabla s)\right] d V,
$$

where $W_{\mathrm{es}}(\mathbf{E}, s)=-\frac{\varepsilon}{2} \mathbf{E} \cdot \mathbf{E}$ is the complementary electrostatic energy per unit volume, $W_{\mathrm{d}}(s)=W_{\mathrm{c}}[1-f(s)]$ is the damage energy with $W_{c}$ representing the critical density of electrostatic energy, and $W_{\mathrm{i}}(\nabla s)=\frac{\Gamma}{4} \nabla s \cdot \nabla s$ is the gradient energy term to regulate sharp phase boundaries. The electric field vector $\mathbf{E}$ is further related to the field of electric potential $\phi(\mathbf{x}, t)$ by $\mathbf{E}=-\nabla \phi$. Notably, the material parameter $\Gamma$ is approximately the breakdown energy. Assuming linear kinetics: $\partial s / \partial t=-m \delta \Pi / \delta s$, the evolution equation for the damage variable $s$ can be obtained after substituting in the detailed forms of the energy functions:

$$
\frac{1}{m} \frac{\partial s}{\partial t}=\frac{\varepsilon^{\prime}(s)}{2} \nabla \phi \cdot \nabla \phi+W_{\mathrm{c}} f^{\prime}(s)+\frac{\Gamma}{2} \nabla^{2} s
$$


Here, mobility $m$ is a material parameter that characterizes the speed of damage evolution in the dielectric solid. By normalizing all length by the characteristic thickness of the damage zone $l=\sqrt{\Gamma / W_{c}}$, energy density by $W_{c}$, time by $1 / m W_{c}$, electric potentials by $\sqrt{\Gamma / \varepsilon_{0}}$, the dimensionless governing equations can be written as:

$$
\begin{gathered}
\bar{\nabla} \cdot\left[\frac{1}{f(s)+\eta} \bar{\nabla} \bar{\phi}\right]=0, \\
\frac{\partial s}{\partial \bar{t}}=-\frac{f^{\prime}(s)}{2[f(s)+\eta]^{2}} \bar{\nabla} \bar{\phi} \cdot \bar{\nabla} \bar{\phi}+f^{\prime}(s)+\frac{1}{2} \bar{\nabla}^{2} s,
\end{gathered}
$$

in which the corresponding dimensionless quantities are symbolized with over-bars. With proper boundary and initial conditions, Eqs. (4) and (5) can be solved for the dimensionless unknown fields $\bar{\phi}(\overline{\mathbf{x}}, \bar{t})$ and $s(\overline{\mathbf{x}}, \bar{t})$. Since the focus of the current paper is mainly on the mechanism study of initiation and propagation of electrical treeing, all simulations are carried out in two-dimensional (2D) domains. To represent the effect of material defects and to introduce randomness in the simulation, we add a small random perturbation with maximum dimensionless magnitude 0.1 to the coefficient of the second term on the right-hand side of Eq. (5), which corresponds to the damage energy.

\section{Results and discussion}

In this section, simulations of the electrical treeing phenomenon in parallel plate capacitors, cylindrical capacitors are individually carried out through the phase-filed model. Composites loaded with fillers of different permittivity, breakdown energy, damage mobility are calculated as well. All calculations in this section are carried out by implementing the differential equations (4) and (5) in COMSOL Multiphysics 5.3a, 
a commercial finite element package.

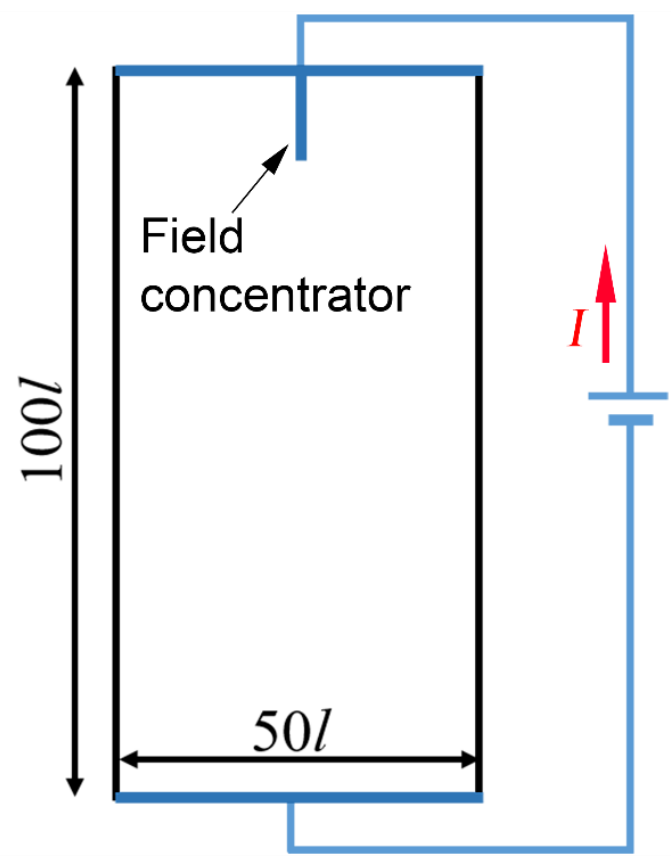

Fig. 1. Schematic of the loading setup of dielectric parallel plate capacitors with a field concentrator.

\subsection{Electrical treeing in homogeneous dielectric parallel plate capacitors}

We first apply our phase-field model in the geometry of a parallel plate capacitor to study the dependence of electrical treeing process on discharge speed. The material is taken to be homogeneous except the random perturbation. The electric voltage is applied via electrodes attached to the top and bottom edges as sketched in Fig. 1. The width and height of this two-dimensional plan-parallel capacitor are $50 \mathrm{l}$ and $100 \mathrm{l}$, respectively. As mentioned above, electrical treeing usually initiates at the points of electric field concentration. Here in our model, a conductive field concentrator is introduced for a controllable field concentration. The voltage $(V)$ between two electrodes is applied by controlling the total charge accumulation $(Q)$ of the bottom electrode, which increases linearly with time, $Q=I t$. Such a loading condition 
corresponds to an ideal current source of constant electric current $I$. In numerical models, a dimensionless discharge speed $\bar{I}$ is taken by normalizing $I$ by $m \Gamma \sqrt{W_{c} \varepsilon_{0}}$. The dimensionless discharge speed measures the discharge speed relative to the rate of material damage.
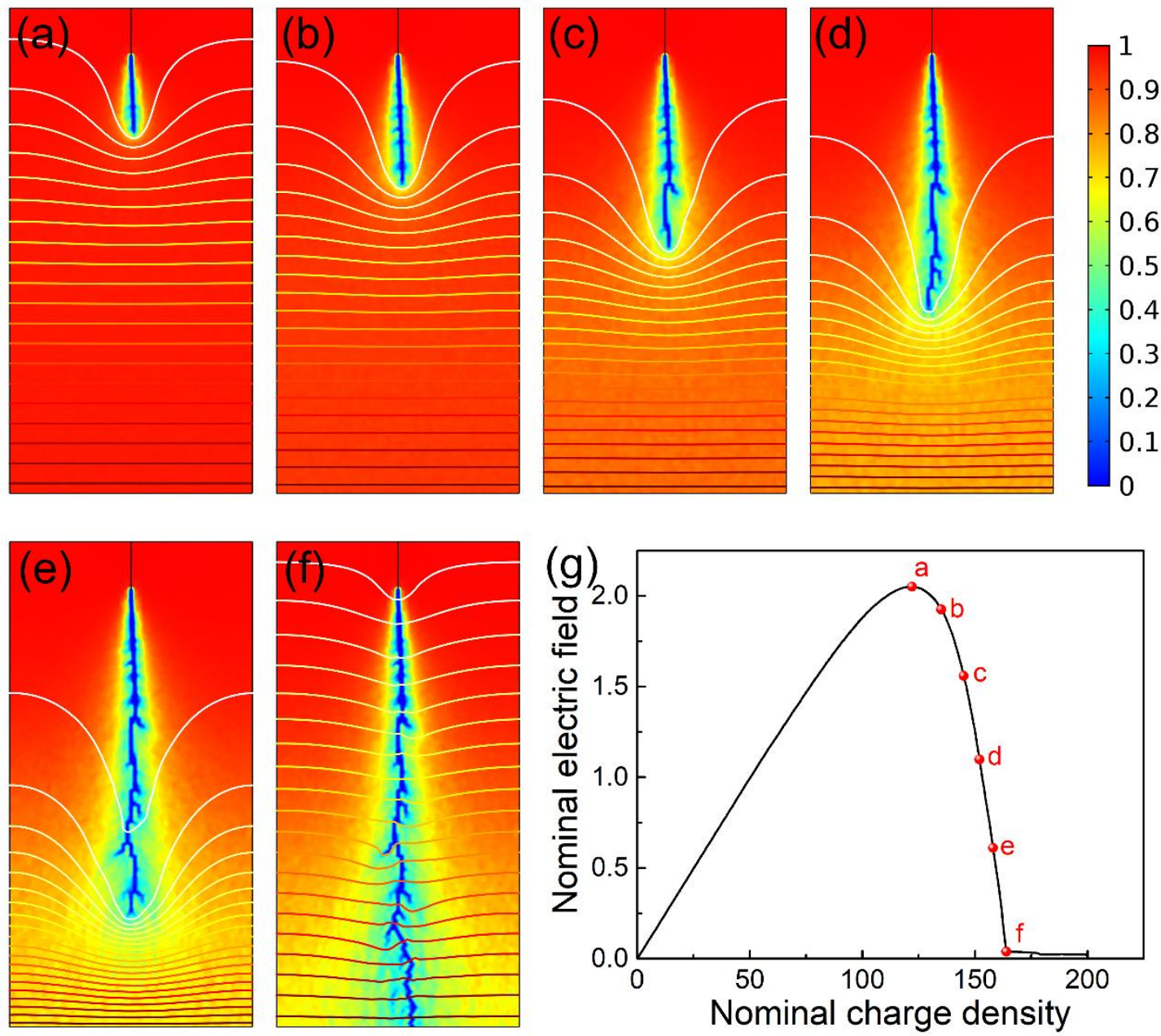

Fig. 2. (a) to (f) Snapshots of the dielectric breakdown process of a dielectric parallel plate capacitor at a dimensionless discharge speed $\bar{I}=1000$, showing the evolution of an electrical tree. The six snapshots (a) through (f) correspond to the six dots on (g). The shading represents the distribution of damage variable $s$. The contour lines are the equipotential lines of the then-current electric field. (The same on all other figures in the remainder of the paper.) (g) The nominal field-charge-density relation associated 
with the process.

The evolution of the electrical tree and the dielectric breakdown process in a parallel plate capacitor under a dimensionless discharge speed, $\bar{I}=1000$, are shown by the six snapshots in Fig. 2 (a) through (f), which correspond to the six points indicated on Fig. $2(\mathrm{~g})$. The effect of the dimensionless discharge speed on electrical treeing will be discussed later. It can be found from Fig. 2 (a) that an electric tree initiates at the tip of the field concentrator and starts to propagate when the applied voltage reaches a critical value. While the main breakdown path is relatively straight, it is usually accompanied by a number of short side branches, which effectively dissipate more energy than a straight path without branches. It is worth noting that, under such a high discharge speed, a large volume in the bulk is partially damaged, especially at the later stage, e.g. Fig. 2(c). The distributed damage also promotes branching. After the gap between the top and bottom electrodes is bridged by the electrical tree, the dielectric capacitor is fully broken-down as seen in Fig. 2 (f). The field-charge-density curve is expected to exhibit two stages and shows clearly in Fig. 2 (g). The dielectric behavior of this parallel plate capacitor is linear below a critical electric field (dot a on Fig. 2 (g)), i.e. the nominal dielectric strength with the presence of the field concentrator. In the second stage, the electrical tree initiates and then propagates, resulting in the decrease in the nominal electric field. Notably, in contrast to the sharp drop in a quasi-static case [50], the drop of the electrical field of this sample is more gradual due to the treeing process. Evidently, the electrical treeing before breakdown is directly related to the degradation of dielectric material. 

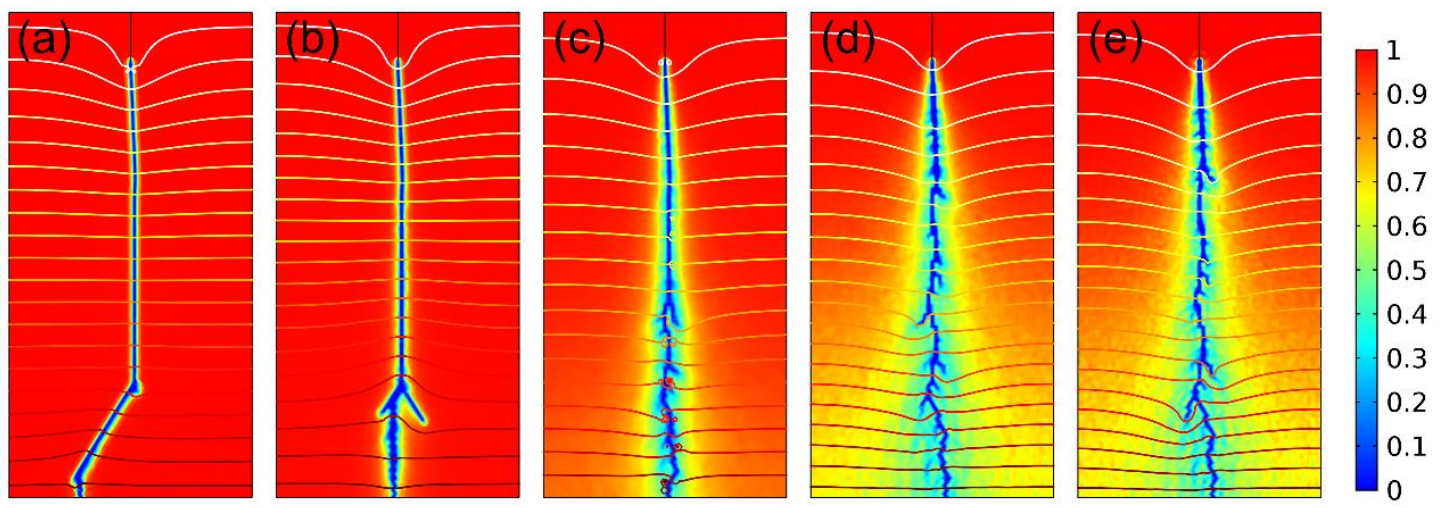

Fig. 3. The final electrical treeing patterns after breakdown under dimensionless discharge speeds of (a) $\bar{I}=1$, (b) $\bar{I}=10$, (c) $\bar{I}=100$, (d) $\bar{I}=1000$, (e) $\bar{I}=10000$. It has been reported that the AC frequency has a large impact on the initiation and propagation of electrical treeing[26, 27], while similar phenomena have also been observed on samples subject to direct current (DC) [14]. In our simulation, we adopt different dimensionless discharge speed $\bar{I}$ to study the mechanism of such effect. The final electrical treeing patterns after the breakdown under different dimensionless discharge speed are plotted in Fig. 3. It is evident that the treeing morphology changes from a branchless straight breakdown path under a lower discharge speed to a multibranched pattern under a higher discharge speed. Collateral damage is also observed in the bulk material in the case of branched treeing. The nominal field-charge-density relation under various dimensionless discharge speed and the relation of nominal breakdown strength against dimensionless discharge speed are displayed in Figs. 4 (a) and (b), respectively. As the dimensionless discharge speed increases, the dielectric material requires a higher charge density to be damaged. More importantly, the higher dimensionless discharge speed, the higher effective breakdown strength the material would exhibit. Under a high dimensionless discharge speed, branches are developed to 
accommodate the larger amount of charge, and the branches and more tortuous breakdown path dissipate more energy, contributing to a high breakdown strength. Such results agree qualitatively with experiments[51-53].

The relative discharge speed $\bar{I}=I / m \Gamma \sqrt{W_{c} \varepsilon_{0}}$ can be changed either by controlling the electric current of the external power source, or by changing the damage mobility $m$, which is a material parameter.
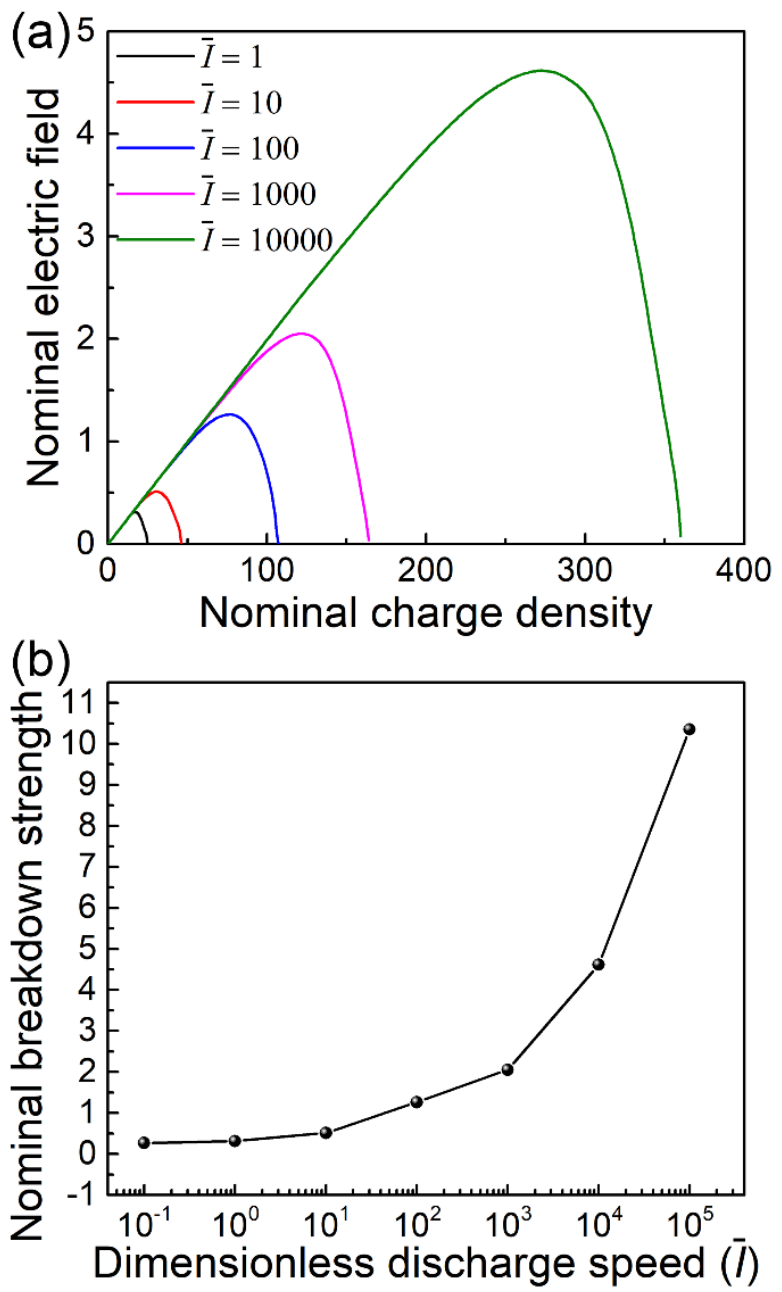

Fig. 4. (a) The nominal field-charge-density relation of parallel plate capacitors under different dimensionless discharge speed. (b) The relation between the nominal breakdown strength and the dimensionless discharge speed. 


\subsection{Electrical treeing in cylindrical capacitors}

The simulations discussed above are carried over the rectangular domain of a parallel plate capacitor. Due to the distribution of local electric field and the introduced field concentrator, there is always one main electrical tree that eventually bridges the top and bottom electrodes when breakdown happens, despite the abundant side branches under a relatively high discharge speed. To study the effect of geometry on treeing morphology, we further simulate the treeing process in a cylindrical capacitor with the cross section sketched in Fig. 5. The radius of the capacitor is taken to be $100 l$, and one of the electrodes is reduced to a thin wire of radius $l$ on the axis of the cylinder. The surface of the cylinder is taken as the other electrode. A voltage $(V)$ is applied between the two electrodes and the sample is loaded by controlling the total charge accumulation $(Q)$ on the outer electrode, similar to the cases described in previous sections.

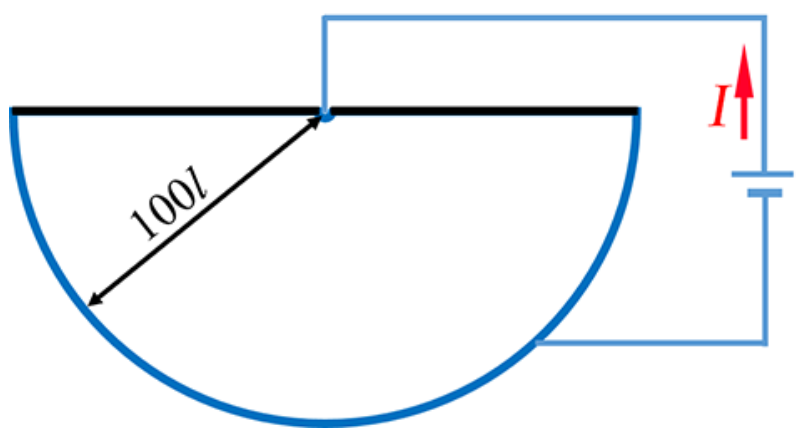

Fig. 5. Schematic drawing of the loading setup of a cylindrical capacitor. Only half of the cross section is modeled, and the electric field is applied between the axial and surface electrodes, with an ideal current source of constant electric current $I$. Symmetry boundary conditions are prescribed on the top surface, except the axial electrode region. 

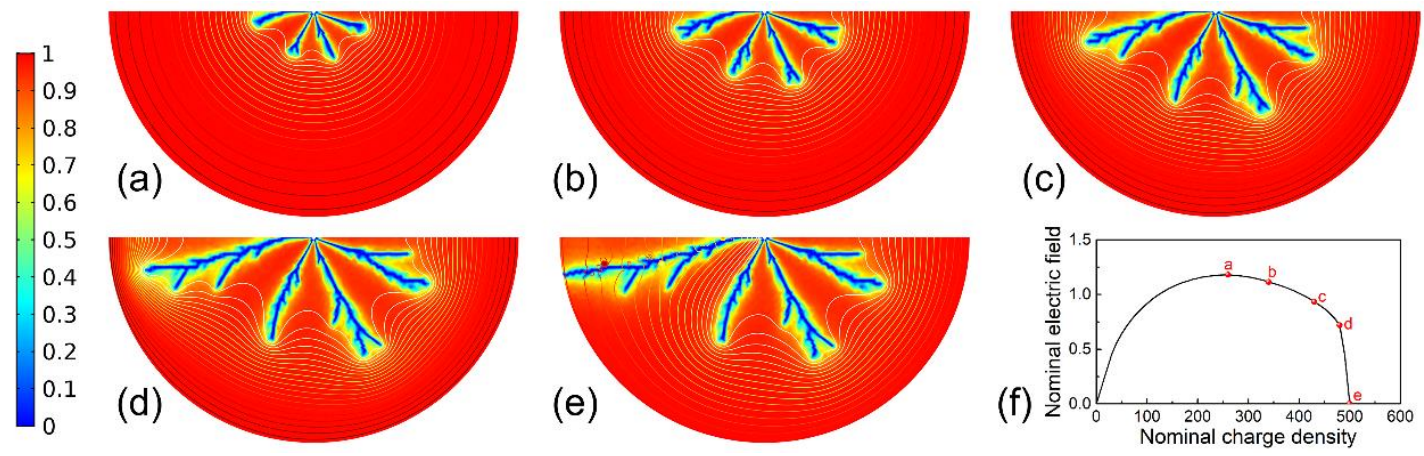

Fig. 6. (a) to (e) Snapshots of electrical treeing and dielectric breakdown in a cylindrical capacitor with dimensionless discharge speed $\bar{I}=1000$. The five snapshots (a) to (e) correspond to the five dots indicated on (f). (f) The nominal field-charge-density relation of the process.

A representative evolution process of electrical treeing and dielectric breakdown in a cylindrical capacitor (dimensionless discharge speed $\bar{I}=1000$ ) is shown by the snapshots in Figs. 6 (a) to (e), corresponding to the five points indicated on Fig. 6 (f). The electrical tree starts around the axial electrode and grows several branches as shown by Figs. 6 (a). From Fig. 6 (b) to Fig. 6 (d), it can be seen that the four main branches propagate separately with short side branches added from time to time. Beyond a critical point, roughly when the distance from a branch tip to the surface electrode equals or becomes smaller than that between neighboring branches, e.g. Fig. 6 (c), the longest branch will dominate and grow much faster than the rest. When it reaches the surface electrode, the two electrodes are short-circuited, and the dielectric material is totally broken down as shown in Fig. 6 (e). The corresponding field-charge-density curve is displayed in Fig. 6 (f) with the time of the snapshots marked. After the linear dielectric regime, the slope gradually decreases due to the partial electrical treeing of the dielectric material. Unlike in a parallel-plate capacitor, the initial damage is stable, 
i.e. a higher voltage can be applied without catastrophic breakdown. From point a to d on Fig. 6 (f), along with the increase of electric charge, the nominal electric field inside the capacitor reduces gradually, corresponding to the reduction in the actual distance between the electrical tree tips and the opposite electrode. The simulation results suggest a strong geometry dependence of breakdown resistance.

To study the effect of discharge speed in the cylindrical geometry, five values of the dimensionless discharge speed $\bar{I}$, varying from 1 to 10000 are adopted in the calculation. The final electrical treeing patterns after breakdown under different discharge speeds are illustrated in Fig. 7. It is quite clear that under relatively low discharge speeds, the electrical treeing patterns tend to have straight paths and fewer branches, while more branched and tortuous breakdown paths are observed on electrical trees formed under relatively high discharge speeds. The later cases dissipate more energy, resulting in a higher effective breakdown strength. These results exhibit the consistent trend as in the cases of parallel-plate capacitors. 

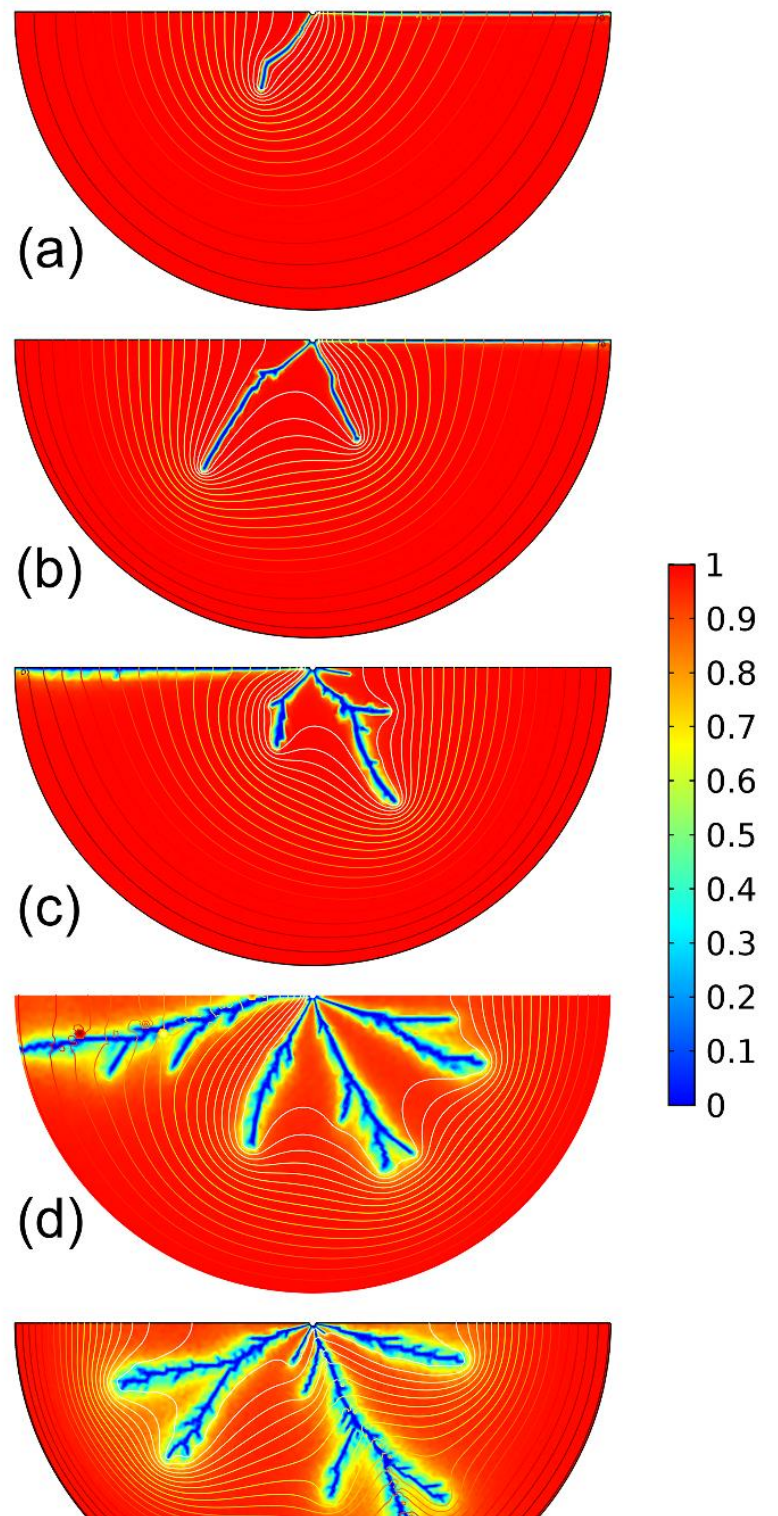

(e)

Fig. 7. The final electrical treeing patterns after breakdown of dielectric materials inside cylindrical capacitors under the dimensionless discharge speed of (a) $\bar{I}=1$, (b) $\bar{I}=$ 10, (c) $\bar{I}=100$, (d) $\bar{I}=1000$, (e) $\bar{I}=10000$. Some branches are located on the top symmetry boundary. 


\subsection{Electrical treeing in dielectric composites filled with fillers}

Electrical treeing is usually the precursor of dielectric breakdown in solids. Naturally, one may enhance the breakdown resistance of dielectrics by suppressing or deflecting an electrical tree, just like the effect of crack deflection in mechanical fracture. Efforts have been made on using fillers to inhibit electrical treeing and the results are encouraging [44]. However, the underlying mechanism of the treeing suppression by fillers is less studied. The current phase-field model enables the numerical study of the mechanisms.
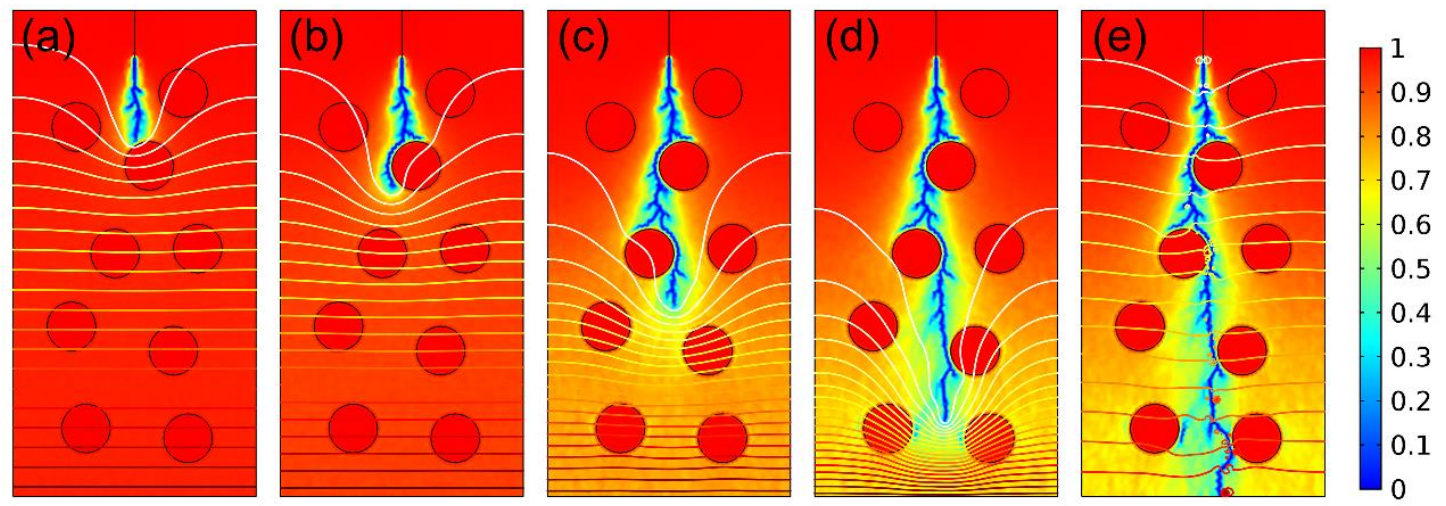

Fig. 8. Snapshots of the electrical treeing process in a composite filled with particles of lower damage mobility. The five snapshots (a) to (e) correspond to the dots on Fig. 9.

In the following numerical examples, particle fillers with different damage mobilities, permittivities, and breakdown energies relative to the matrix will be studied individually. First, the particle fillers that have lower damage mobility, $m_{\mathrm{f}}$, relative to that of the matrix, $m,\left(m_{\mathrm{f}} / m=0.1\right)$, and high damage mobility $\left(m_{\mathrm{f}} / m=10\right)$ are calculated using the present model, and all other parameters are taken to be identical between the filler and the matrix. The relative rate of discharge is taken to be $\bar{I}=1000$. The detailed evolution of electrical trees in these two composites are shown in Fig. 8 and 
Fig. 10. The electrical treeing patterns of these two composites differ drastically. For filler particles with lower mobility, as in Fig. 8 (a) to (e), the electrical tree is deflected at the particle surface and branches. Evidently, the particles with low damage mobility (more damage resistive) are quite effective at suppressing or delaying electrical tree propagation. The dielectric breakdown strength is enhanced by up to $\sim 30 \%$ compared with that without fillers (Fig. 2).

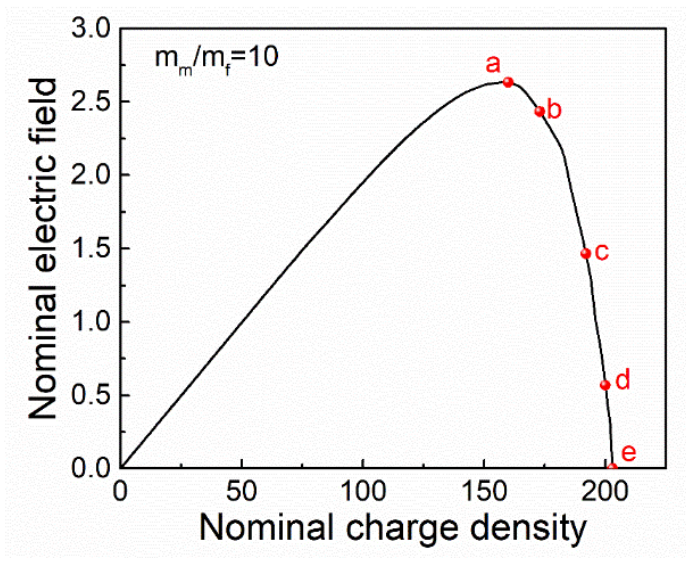

Fig. 9. The nominal field-charge-density relation of a dielectric composite filled with particles of lower damage mobility.

In the case that particle fillers have higher damage mobility than the matrix, the electrical treeing pattern is completely different from that in Fig. 10. The treeing process undergoes two stages. First, electrical trees initiate in each individual filler particles which subsequently breakdown due to the high damage mobility, as in Fig. 10 (a). Afterwards, the breakdown paths in neighboring particles are bridged in between, leading to the final breakdown of the composite. In this stage, although the postbreakdown particles act as field concentrators, due to the finite radius and the spatial distribution of particles, the field concentration is not as significant as that induced by 
the tip of a breakdown path. As a result, the degradation in the material is more stable than a homogeneous material, and the field-charge-density curve exhibits multiple steps (Fig. 11), each corresponding to a bridging event between two particles, although the effective breakdown strength is lower.
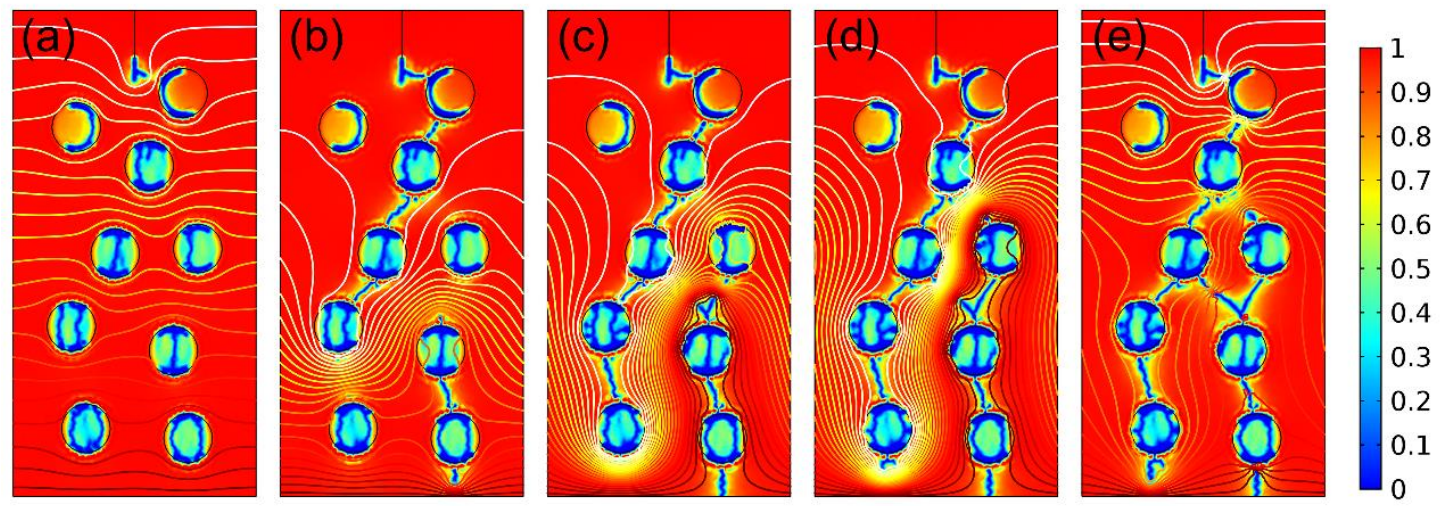

Fig. 10. Snapshots of electrical treeing in a dielectric composite filled with particles of higher damage mobility. The snapshots (a) to (e) correspond to the dots on the fieldcharge-density curve in Fig. 11.

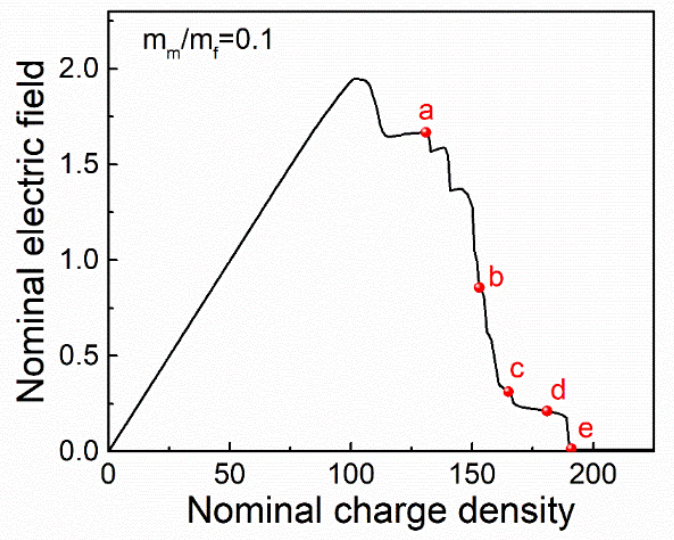

Fig. 11. The nominal field-charge-density relation of a dielectric composite filled with particles of higher damage mobility.

For comparison, composites filled with particles of different permittivities and breakdown energies are also calculated. The final electrical treeing patterns after full breakdown of all cases are shown in Fig. 12, and nominal breakdown strengths of all 
cases extracted from field-charge-density relation are summarized in Table 1, together with the parameter combinations. All these cases are loaded by controlling the dimensionless discharge speed at $\bar{I}=1000$. As shown by Fig. 12(a), when a dielectric is filled with particles of lower permittivity, partial damage and electrical trees tend to nucleate near the lateral sides of the particles, where the electric field is concentrated due to permittivity difference. These local damage zones will then be connected in a stage of higher nominal electric field, leading to the ultimate breakdown of the composite. This type of composite demonstrates a $40 \%$ higher effective breakdown strength due to the distributed damage near all filler particles and the delocalization of the electric field. In practice, silicon dioxide and other low permittivity fillers are often used in polymer matrices (e.g. PVDF) to enhance the breakdown strength and thus increase the energy density $[16,54]$. Voids inside a polymer matrix may also be seen as low permittivity fillers, but with relatively low breakdown energy, they rarely enhance the breakdown strength, as will be discussed later. Naturally, we can imagine that a dielectric composite loaded with particles of higher permittivity would in turn worsen the breakdown resistance. Indeed, the field concentration near the two ends of a particle along the direction of applied field would direct the electric treeing towards a straighter path with fewer branches, resulting in a low effective breakdown strength.

Table 1. The nominal breakdown strength (NBS) of dielectric composites filled with particles of various damage mobilities $\left(m_{\mathrm{f}} / m\right)$, permittivities $\left(\varepsilon_{\mathrm{f}} / \varepsilon\right)$ and breakdown energy $\left(\Gamma_{\mathrm{f}} / \Gamma\right)$. The corresponding electrical treeing patterns (ETP) are also listed. 


\begin{tabular}{ccccc}
\hline$m_{\mathrm{f}} / m$ & $\varepsilon_{\mathrm{f}} / \varepsilon$ & $\Gamma_{\mathrm{f}} / \Gamma$ & NBS & ETP \\
\hline 1 & 1 & 1 & 2.05 & Fig. 2 \\
1 & 0.1 & 1 & 2.92 & Fig. 12(a) \\
1 & 10 & 1 & 2.00 & Fig. 12(b) \\
1 & 1 & 0.1 & 2.61 & Fig. 12(c) \\
1 & 10 & 0.1 & 2.01 & Fig. 12(d) \\
1 & 1 & 10 & 2.63 & Fig. 12(e) \\
10 & 1 & 1 & 1.95 & Fig. 10 \\
0.1 & 1 & 1 & 2.63 & Fig. 8 \\
\hline
\end{tabular}

Figure 12(c) shows the effect of filler particles with lower breakdown energy, which break down prior to the matrix and act as the sacrificial phase. The electrical tree in the matrix will then grow by connecting these broken-down particles in the next stage. Although the phenomenon is similar as that in the composite of particles with higher damage mobility, the consequences are significantly different: fillers of higher damage mobility accelerate the speed of electrical treeing propagation, resulting in a lower overall breakdown strength; while the fillers of lower breakdown energy only distribute the damage and relieve field concentration without affecting the breakdown speed, leading to a relatively high breakdown strength.
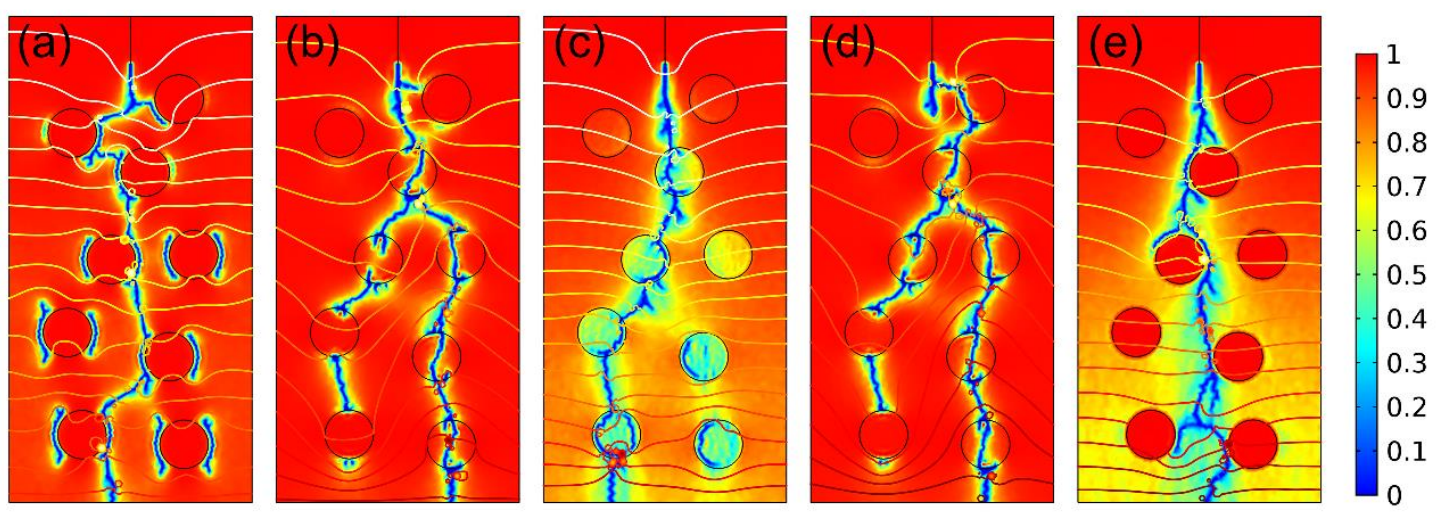

Fig. 12. The final electrical treeing patterns after breakdown of dielectric composites

filled with particles of (a) lower permittivity, (b) higher permittivity, (c) lower 
breakdown energy, (d) higher permittivity and lower breakdown energy, (e) higher breakdown energy.

To compare between the effects of filler permittivity and breakdown energy, we further simulate the case when the filler particles have higher permittivity but lower breakdown energy. As shown by Fig. 12(d), the final treeing pattern is quite similar to that of high filler permittivity only, as in Fig. 12 (b). Not only the treeing morphologies look similar, the effective breakdown strengths are also equally low. Despite the low breakdown energy of the filler material, the field concentration directs the electrical trees through the particles and causes localized damage, preventing the sacrificial mechanism from being effective. It is similar to the case in commonly used energystorage polymer-ceramic composites, which use ceramic fillers (e.g. barium titanate and strontium titanate) possessing high permittivity and low breakdown energy [55-57]. In these composites, the effective breakdown strength is decreased to some extent, but the effective permittivity can be significantly enhanced, at last resulting in a relatively high energy-storage density.

Interestingly, enhancement is also seen in a dielectric composite with fillers of higher breakdown energy. Instead of acting as the sacrificial phase, these filler particles do not breakdown prior to or together with the matrix. Forming a barrier to rest and deflect the electrical trees, these particles promote branching and lead to a tortuous treeing morphology, and ultimately yield a higher effective breakdown strength of the composite.

In summary, fillers may enhance the breakdown resistance of dielectrics through 
changing the morphology of treeing patterns. The more branches, the less sensitive to field concentration, and the higher the overall breakdown strength. The phase-field simulation results suggest that fillers of lower permittivity, higher breakdown energy or lower damage mobility are effective in suppressing tree propagation and/or promoting branching, therefore resisting catastrophic breakdown and effectively enhancing the strength when field concentration is inevitable.

\section{Conclusion}

In this work, a phase-field model is developed to simulate the initiation and propagation of electrical trees in dielectric solids of arbitrary geometries. In a parallel plate capacitor containing homogeneous dielectrics, it is found that the discharge speed relative to the rate of degradation in the material plays an important role in the treeing morphology as well as the breakdown strength. Higher discharge speed leads to a fractal shaped electrical tree with more side branches and a tortuous breakdown path. The formation of the longer breakdown path with branches can dissipate more energy and therefore enhance the breakdown resistance of dielectrics. With a symmetric geometry that stabilizes treeing, the phenomenon is more clearly seen in a cylindrical capacitor. At a relatively high discharge speed, antler-like treeing patterns originated from the axial electrode are observed in a cylindrical capacitor. Further, the effects of fillers of various material properties on electric treeing are also studied by using the phase-field model. Numerical results suggest that fillers of lower permittivity, higher breakdown energy or lower damage mobility will promote fractal treeing and thus resist 
catastrophic breakdown. As a result, the effective breakdown strength of these composites with the presence of field concentrators can be enhanced. The model not only provides a mechanistic understanding towards the morphology of electrical treeing phenomena, but may also serve as guidance towards the design of breakdown-resistant high-voltage dielectric composites.

\section{Acknowledgments}

$\mathrm{ZC}$ is grateful to the financial support from Tsinghua University on his visit at Iowa State University, and to Iowa State University for hosting the visit. XW acknowledges the supported from Ministry of Sciences and Technology of China through National Basic Research Program of China (973 Program 2015CB654604), National Natural Science Foundation of China (Grant No. 51672148), and CBMI Construction Co., Ltd..

\section{References:}

[1] Yao Z, Song Z, Hao H, Yu Z, Cao M, Zhang S, et al. Homogeneous/InhomogeneousStructured Dielectrics and their Energy-Storage Performances. Adv Mater 2017.

[2] Hao Y, Wang X, Bi K, Zhang J, Huang Y, Wu L, et al. Significantly enhanced energy storage performance promoted by ultimate sized ferroelectric $\mathrm{BaTiO}_{3}$ fillers in nanocomposite films. Nano Energy 2017;31:49-56.

[3] Zhang X, Shen Y, Xu B, Zhang Q, Gu L, Jiang J, et al. Giant Energy Density and Improved Discharge Efficiency of Solution-Processed Polymer Nanocomposites for Dielectric Energy Storage. Advanced Materials 2016. 
[4] Wang Y, Cui J, Yuan Q, Niu Y, Bai Y, Wang H. Significantly Enhanced Breakdown Strength and Energy Density in Sandwich-Structured Barium Titanate/Poly(vinylidene fluoride) Nanocomposites. Adv Mater 2015;27:6658-63.

[5] Chen Q, Shen Y, Zhang S, Zhang QM. Polymer-Based Dielectrics with High Energy Storage Density. Annual Review of Materials Research 2015;45:433-58.

[6] Wang Y, Hou Y, Deng Y. Effects of interfaces between adjacent layers on breakdown strength and energy density in sandwich-structured polymer composites. Composites Science and Technology 2017;145:71-7.

[7] Zheng M-S, Zha J-W, Yang Y, Han P, Hu C-H, Dang Z-M. Enhanced breakdown strength of poly(vinylidene fluoride) utilizing rubber nanoparticles for energy storage application. Applied Physics Letters 2016;109.

[8] Hildinger T, Hydro V, Gröppel BP, Weil M, Weidner JR. Improved generator performance with a nanocomposite high voltage insulation system for stator windingsA status report. Cigre Working Group A 2016;1:109.

[9] Kimoto T. Material science and device physics in SiC technology for high-voltage power devices. Jpn J Appl Phys 2015;54:040103.

[10] Cai Z, Wang X, Luo B, Hong W, Wu L, Li L. Multiscale design of high-voltage multilayer energy-storage ceramic capacitors. Journal of the American Ceramic Society 2018;101:1607-15.

[11] Zhang X, Shen Y, Zhang Q, Gu L, Hu Y, Du J, et al. Ultrahigh energy density of polymer nanocomposites containing BaTiO3@TiO2 nanofibers by atomic-scale interface engineering. Advanced Materials 2015;27:819-24. 
[12] Han K, Li Q, Chanthad C, Gadinski MR, Zhang G, Wang Q. A Hybrid Material Approach Toward Solution-Processable Dielectrics Exhibiting Enhanced Breakdown Strength and High Energy Density. Advanced Functional Materials 2015;25:3505-13.

[13] Li Q, Han K, Gadinski MR, Zhang G, Wang Q. High Energy and Power Density Capacitors from Solution-Processed Ternary Ferroelectric Polymer Nanocomposites. Advanced Materials 2014;26:6244-9.

[14] Pallon LK, Nilsson F, Yu S, Liu D, Diaz A, Holler M, et al. Three-Dimensional Nanometer Features of Direct Current Electrical Trees in Low-Density Polyethylene. Nano Lett 2017;17:1402-8.

[15] Nyanteh Y, Graber L, Edrington C, Srivastava S, Cartes D. Overview of simulation models for partial discharge and electrical treeing to determine feasibility for estimation of remaining life of machine insulation systems. Electrical Insulation Conference (EIC), 2011: IEEE; 2011. p. 327-32.

[16] Ahmad MH, Bashir N, Ahmad H, Abd Jamil AA, Suleiman AA. An Overview of Electrical Tree Growth in Solid Insulating Material with Emphasis of Influencing Factors, Mathematical Models and Tree Suppression. TELKOMNIKA Indonesian Journal of Electrical Engineering 2014;12.

[17] Dissado LA. Understanding electrical trees in solids: from experiment to theory. IEEE Trns Dielectr Electr Insul 2002;9:483-97.

[18] Shimizu N, Laurent C. Electrical tree initiation. IEEE Trns Dielectr Electr Insul 1998;5:651-9.

[19] Barclay A, Stevens G. Statistical and fractal characteristics of simulated electrical 
tree growth. Dielectric Materials, Measurements and Applications, 1992, Sixth International Conference on: IET; 1992. p. 17-20.

[20] Zhou Y, Liu R, Hou F, Zhang X, Xue W. Morphology of electrical trees in silicon rubber. Journal of Electrostatics 2013;71:440-8.

[21] Vaughan A, Dodd SJ, Sutton S. A Raman microprobe study of electrical treeing in polyethylene. Journal of materials science 2004;39:181-91.

[22] Anil Kumar CR, Deepa S, Mishra AK, Sarathi R. Investigation into the failure of XLPE cables due to electrical treeing: a physico chemical approach. Polymer Testing $2003 ; 22: 313-8$

[23] Champion J, Dodd S. The effect of voltage and material age on the electrical tree growth and breakdown characteristics of epoxy resins. Journal of Physics D: Applied Physics 1995;28:398.

[24] Zhang Y, Zhou Y, Zhang L, Lin Z, Liu J, Zhou Z. Electrical treeing behaviors in silicone rubber under an impulse voltage considering high temperature. Plasma Science and Technology 2018;20:054012.

[25] Du B, Ma Z, Gao Y, Han T. Effect of ambient temperature on electrical treeing characteristics in silicone rubber. IEEE Trns Dielectr Electr Insul 2011;18.

[26] Nie Q, Zhou Y, Chen Z, Chen H. Effect of frequency on electrical tree characteristics in silicone rubber. Properties and Applications of Dielectric Materials, 2009 ICPADM 2009 IEEE 9th International Conference on the: IEEE; 2009. p. 513-6. [27] Chen G, Tham C. Electrical treeing characteristics in XLPE power cable insulation in frequency range between 20 and $500 \mathrm{~Hz}$. IEEE Trns Dielectr Electr Insul 
2009;16:179-88.

[28] Hauschild W, Mosch W. Statistical techniques for high-voltage engineering: IET; 1992.

[29] Bahadoorsingha $\Psi$ S, Sambeharryb S, Balliramc R, Sharmad C. Electrical Treeing Diagnostics-An Approach Combining Optical Measurements and Partial Discharge Statistics. 2012.

[30] Kottegoda N. Fitting Johnson Sb curve by the method of maximum likelihood to annual maximum daily rainfalls. Water Resources Research 1987;23:728-32.

[31] Weibull W. A STATISTICAL DISTRIBUTION FUNCTION OF WIDE APPLICABILITY. J Appl Mech-Trans ASME 1951;18:293-7.

[32] Niemeyer L, Pietronero L, Wiesmann HJ. Fractal Dimension of Dielectric Breakdown. Physical Review Letters 1984;52:1033-6.

[33] Dissado L, Sweeney P, Fothergill J. The field dependence of electrical tree growth. Dielectric Materials, Measurements and Applications, 1992, Sixth International Conference on: IET; 1992. p. 13-6.

[34] Champion J, Dodd S, Stevens G. Analysis and modelling of electrical tree growth in synthetic resins over a wide range of stressing voltage. Journal of Physics D: Applied Physics 1994;27:1020.

[35] Dissado LA, Fothergill JC. Electrical degradation and breakdown in polymers: IET; 1992.

[36] Hofacker M, Miehe C. A phase field model of dynamic fracture: Robust field updates for the analysis of complex crack patterns. International Journal for Numerical 
Methods in Engineering 2013;93:276-301.

[37] Borden MJ, Verhoosel CV, Scott MA, Hughes TJR, Landis CM. A phase-field description of dynamic brittle fracture. Computer Methods in Applied Mechanics and Engineering 2012;217-220:77-95.

[38] Spatschek R, Brener E, Karma A. Phase field modeling of crack propagation. Philosophical Magazine 2011;91:75-95.

[39] Hakim V, Karma A. Laws of crack motion and phase-field models of fracture. Journal of the Mechanics and Physics of Solids 2009;57:342-68.

[40] Zhang B, Li Z, Ren M, Liu J, Moran T, Huey B, et al. A superior nanolaminate dielectric barrier coating for high breakdown strength. Electrical Insulation and Dielectric Phenomenon (CEIDP), 2017 IEEE Conference on: IEEE; 2017. p. 461-4.

[41] Cai Z, Wang X, Luo B, Li L. Hierarchical-structured dielectric permittivity and breakdown performances of polymer-ceramic nanocomposites. Ceram Int 2018;44:843-8.

[42] Cai Z, Wang X, Luo B, Hong W, Wu L, Li L. Nanocomposites with enhanced dielectric permittivity and breakdown strength by microstructure design of nanofillers. Composites Science and Technology 2017;151:109-14.

[43] Chen S-s, Hu J, Gao L, Zhou Y, Peng S-m, He J-1, et al. Enhanced breakdown strength and energy density in PVDF nanocomposites with functionalized $\mathrm{MgO}$ nanoparticles. RSC Advances 2016;6:33599-605.

[44] Musa FN, Bashir N, Ahmad MH, Buntat Z. Electrical treeing in high voltage insulations: a review on nanocomposite insulating materials and their processing 
techniques. JOURNAL OF OPTOELECTRONICS AND ADVANCED MATERIALS 2015;17:462-76.

[45] Sridhar A, Thomas MJ. Electrical treeing in polyethylene: effect of nano fillers on tree inception and growth. High Voltage Engineering and Application (ICHVE), 2010 International Conference on: IEEE; 2010. p. 576-9.

[46] Pitsa D, Vardakis G, Danikas M, Kozako M. Electrical Treeing Propagation in Nanocomposites and the Role of Nanofillers: Simulationwith the Aid of Cellular Automata. Journal of Electrical Engineering 2010;61.

[47] Danikas MG, Tanaka T. Nanocomposites-a review of electrical treeing and breakdown. IEEE Electrical Insulation Magazine 2009;25:19-25.

[48] Cai Z, Wang X, Luo B, Hong W, Wu L, Li L. Dielectric response and breakdown behavior of polymer-ceramic nanocomposites: The effect of nanoparticle distribution. Composites Science and Technology 2017;145:105-13.

[49] Hong W, Pitike KC. Modeling Breakdown-resistant Composite Dielectrics. Procedia IUTAM 2015;12:73-82.

[50] Pitike KC, Hong W. Phase-field model for dielectric breakdown in solids. Journal of Applied Physics 2014;115:8.

[51] Miyairi K. Frequency-Dependent Dielectric Breakdown in Thin Polyvinylcarbazole Films. Jpn J Appl Phys 2003;42:5153-7.

[52] Miyairi K. The frequency dependent dielectric breakdown in some thin polymers. Properties and Applications of Dielectric Materials, 2003 Proceedings of the 7th International Conference on: IEEE; 2003. p. 949-52. 
[53] Miyairi K, Itoh E. Influence of ionic impurities on the AC breakdown and its frequency dependence in the polymer thin film. Solid Dielectrics, 2001 ICSD'01 Proceedings of the 2001 IEEE 7th International Conference on: IEEE; 2001. p. 155-8. [54] Han B, Jiao M, Li C, Zhang C, Wu Z, Wang Y, et al. QM/MD simulations on the role of $\mathrm{SiO} 2$ in polymeric insulation materials. RSC Advances 2016;6:555-62.

[55] Hou Y, Deng Y, Wang Y, Gao H. Uniform distribution of low content BaTiO3 nanoparticles in poly(vinylidene fluoride) nanocomposite: toward high dielectric breakdown strength and energy storage density. RSC Advances 2015;5:72090-8.

[56] Niu Y, Yu K, Bai Y, Xiang F, Wang H. Fluorocarboxylic acid-modified barium titanate/poly(vinylidene fluoride) composite with significantly enhanced breakdown strength and high energy density. RSC Advances 2015;5:64596-603.

[57] Zhang Q, Gao F, Zhang C, Wang L, Wang M, Qin M, et al. Enhanced dielectric tunability of $\mathrm{Ba} 0.6 \mathrm{Sr} 0.4 \mathrm{TiO} 3 / \mathrm{Poly}$ (vinylidene fluoride) composites via interface modification by silane coupling agent. Composites Science and Technology 2016;129:93-100. 